\title{
Addendum
}

\section{Kinetics of Oxidation of Ferrous Alloys by Superheated Steam*}

Nicholas J. Cory and Thelma M. Herrington

\section{The Location of Hydrogen in the Kinetics of Oxidation of Ferrous Alloys by Superheated Steam $\dagger$}

Nicholas J. Cory and Thelma M. Herrington

The work described in the two papers listed above was supported by the Harwell laboratory of the AKAEA under a CASE arrangement with Reading University; the funding was provided by SERC under the terms of the CASE award scheme with a financial contribution from the underlying program of the UKAEA. 